\title{
Kritische Betrachtungen über die Fiehe'sche Reaktion.
}

\author{
Von
}

\section{Martin Klassert.}

Mitteilung aus dem Handelslaboratorium Dr. Wilhelm Hoepfner in Hamburg.

\section{[Eingegangen am 24. Dezember 1908.]}

Beim Bekanntwerden der Fiehe'schen Reaktion waren wohl sämtliche Honigchemiker etwas zweifelhaft über den Wert der Reaktion; hatten sich doch schon so manche anfänglich sehr aussichtsreich erscheinende Neuerungen auf dem Gebiete der Honiganalyse als wertlos erwiesen infolge der besonderen Natur des Honigs, der nach der Herkunft und der Art seiner Gewinnung sich verschieden verhält.

Dann mehrten sich, und zwar viel rascher als seinerzeit bei der Ley'schen Reaktion, die Stimmen, die der Fiebe'schen Reaktion eine mehr oder weniger große Be. deutung für die Honiganalyse zuschrieben. Eines der bekanntesten mit der Untersucbung von Honig beschäftigten Laboratorien begnügte sich sogar mit der Feststellung von Aussehen, Geruch, Geschmack, Polarisation und der Fiehe'schen Reaktion, um den Honig als "gefälscht" zu bezeichnen und zwar wesentlich auf Grund einer schwach, aber deutlich positiven Fiebe'schen Reaktion, die eine "Zumischung von künstlichem Invertzucker" anzeige.

Gerade dieser Fall war es, der mich auf eine mögliche Unzuverlässigkeit der Reaktion brachte, nachdem ich bis dahin sehr befriedigende Ergebnisse bei der Anwendung der Fiehe'schen und der Ley'schen Reaktion erhalten hatte.

Die Untersuchung einer Probe Honig - Importware - hatte analytisch normale Werte ergeben; die Ley'sche Reaktion verlief ebenfalls normal, die Fiehe'sche Reaktion ergab die bekannte "eben wahrnehmbare, rasch verschwindende Rosafärbung“, sodaß zu einer Verdächtigung keinerlei Anlaß vorlag. Nach Verkauf des Honigs wurde er auf Grund des oben erwähnten Gutachtens dem Verkäufer als verfälscht zur Verfügung gestellt.

Die Reellität des Importeurs anzuzweifeln, lag kein Grund vor; die untersuchten Proben erwiesen sich als identisch, nur zeigte die Probe des Empfängers deutliche, die des Verkäufers keine Fiehe'sche Reaktion.

Die Vermutung, daß die Proben des Empfängers dem schon gereinigten Honig (umgeschmolzen und von gröberen Verunreinigungen getrennt) entstammten, bestätigte sich, und die daraufhin erst mit einigen Wabenhonigen, dann mit einer größeren Anzahl von Importhonigen angestellten Versuche lieferten ein sehr überraschendes Ergebnis.

Während schon diese Untersuchungen über den Einfluß der Erwärmung auf die Fiehe'sche Reaktion im Gange waren, erschienen verschiedene darauf bezügliche Notizen und Abhandlungen, zunächst ein Bericht Fiehe's über eine Abänderung seines Verfahrens ${ }^{\text {t) }}$.

Das Referat der Pharm. Zentralhalle ${ }^{2}$ ) über die Fiehe'sche Reaktion erwähnte die von Fiehe als unwesentlich bezeichneten, bei manchen reinen Honigen beob-

1) Chem.ZZtg. 1908, 32, 1045.

2) Pharmaz. Zentralhalle 1908, 49, 904. 
achteten, rasch vorübergehenden Rosafärbungen - Fieh e schreibt sie minimalen, in die Ätherlösung übergehenden Fructosemengen zu - und brachte zum Schluß die Bemerkung der Schriftleitung: "Das Erhitzen des Honigs dürfte auch eine Rolle spielen". Fast gleichzeitig damit veröffentlichte $\mathrm{Draw} \mathrm{e}^{1}$ ) einen kurzen Bericht über intensives Eintreten der Reaktion bei Honigen, die im Wasserbad erwärmt waren; dann folgte die Abwehr Fiehe's ${ }^{2}$ ) und endlich die Notiz v. Raumer's ${ }^{3}$ ).

Die ersterwähnte Veröffentlichung Fi e h e's, die eine für mich neue Ausführungsart der Reaktion - Überschichten der Ätherlösung über das Resorcin-Salzsäure-Reagens angab, veranlaßte mich, auch diese noch nachzuprüfen.

Meine Prüfungen erstreckten sich auf 4 Wahenhonige, 1 deutsehen Imkerhonig, und 10 Importhonige, und zwar 2 kalifornische, 2 chilenische, 1 Domingo-, 2 Havana-, 1 Jamaika- und 2 Mexiko-Honige.

Die Ergebnisse waren kurz zusammengefabt folgende:

Der Ausfall der Ley'schen Reaktion wurde durch längeres Erwärmen reiner Honige auf $100^{\circ}$ oder darunter nicht wesentlich geändert.

Die Fiehe'sche Reaktion dagegen warde bei drei von den vier selbst ausgepreßten. Wabenhonigen schon durch Einsetzen der Honige in ein Wasserbad von $90^{\circ} \mathrm{C}$ und Erkaltenlassen so stark hervorgerufen, daß man die Reaktion als ausgesprochen positiv bezeichnen multe. Die direkte Prüfung der Honige ergab keine Spur einer Rotfärbung.

Durch die Bestimmung des Säuregehaltes wurde eine mögliche Ursache des verschiedenen Verbaltens der Honige aufgedeckt: Der gegen das Erwärmen auf $90^{\circ}$ unempfindliche Honig enthielt nur $0,07 \%$, die drei anderen aber enthielten 0,18 , 0,21 bezw. $0,23 \%$ freie Säure, berechnet als Ameisensäure. Nach 1/2-stündigem Erwärmen im siedenden Wasserbade blieb bei dem säurearmen Wabenhonig die Fieh e'sche Reaktion noch immer aus, während sie bei den säurereichen Proben stark, aber entsprechend ihrem verschiedenen Säuregehalt verschieden stark eintrat. Nach 1-stündigem Erwärmen im siedenden Wasser endlich trat auch in dem säurearmen Honig die Fiehe'sche Reaktion ein, zwar nur schwach, aber deutlich. Der deutsche Imkerhonig verhielt sich ebenso wie der säurearme Wabenhonig.

Von den 10 Importhonigen gaben zwei ohne weiteres die Fi ehe'sche Reaktion sehr kräftig (1 Jamaika- und 1 Mexikohonig), zwei ergaben keine Färbung (Chileund Domingo-Honig), alle übrigen gaben mehr oder weniger starke Rosa- bezw. Hellrotfärbung.

Durch das Erwärmen der Honige im Wasserbad verstärkten sich die bei der Fiehe'schen Reaktion entstehenden Färbungen durchweg, auch die beiden ursprünglich nicht reagierenden Honige ergaben nach 1/2-stündigem Verweilen im siedenden Wasserbad ziemlich starke Fiehe'sche Reaktionen, demgegenüber fiel die Ley'sche Reaktion bei allen 10 Proben durchaus normal aus.

Die Unverfälschtheit der 10 Proben nur auf Grund der normalen Ley'schen Reaktion ohne Rücksicht auf den Ausfall der Fiehe'sehen Reaktion behaupten zu wollen, geht natürlicn zu weit, aber nach den oben angeführten Versuchsergebnissen kann ich die Fiehe'sche Reaktion bei ausländischen Honigen durchaus nicht mehr

1) Zeitschr. öffentl. Chem. 1908, 14, 352.

2) Chem.-Ztg. 1908, 32, 1090.

3) Diese Zeitschrift 1908, 16, 817 . 
als beweiskräftig ansehen, da die Honige vielfach äußerst primitiv gewonnen werden und zum Teile sogar auf freier Flamme ausgeschmolzen werden.

Bei inländischen Honigen dürfte sie aber immerhin manchmal nützlich sein, da, wie Fiehe mit Recht betont, die Honigproduzenten und Händler wohl wissen, daß das Erwärmen des Honigs ihn qualitativ nicht verbessert und reiner einheimischer Schleuderhonig selten so fest wird, daß er umgeschmolzen werden muß.

Aber selbst hier mulu man doch immer mit der Möglichkeit der Erwärmung: rechnen, und die etwa positiv ausfallende Fiehe'sche Reaktion ist zunächst nur wie auch Drawe betont - beweisend dafür, daß die untersuchte Probe ganz oder zum Teil auf höhere Temperaturen erwärmt gewesen ist.

$\mathrm{Zu}$ weitergehenden Schlüssen bedarf es ${ }^{-}$heute unbedingt einer vollständigen Analyse, bei der manchmal auch der Stickstoff- und Phosphorsäuregehalt mit herangezogen werden mul.

\title{
Albuminate im Naturhonig und Kunsthonig.
}

\author{
Von
}

\section{R. Lund.}

Vorlänige Mitteilung aus dem Laboratorium von Prof. Dr. Schaffer in Bern.

[Eingegangen am 18. Januar 1909.]

Über den Gehalt des Honigs an Stickstoffsubstanz findet sich in der Fachliteratur ein ziemlich umfangreiches Zahlenmaterial. Die Schwankungen dieses Gehaltes sind bedeutend. J. König ${ }^{1}$ ) gibt für Naturhonige einen Gehalt von $0,03-2,67^{0 / 0}$, im Durchschnitt $1,42 \%$ Stickstoffsubstanz an. Über den Gehalt der Kunsthonige an Stickstoffsubstanz liegen genauere Angaben nicht vor ${ }^{2}$ ).

Da nach den Untersuchungen von $\mathrm{v}$. Planta ${ }^{3}$ ) die Nektararten frei von Stickstoffsubstanz zu sein scheinen, so ist anzunehmen, daß diese wenigstens vorwiegend erst im Honigmagen oder überhaupt im Organismus der Bienen in den Honig hineingelangt. Es schien nun von Interesse zu sein, nicht nur die Zahl der Bestimmungen der gesamten Stickstoffsubstanz in authentischen Honigproben zu vermehren, sondern auch Versuche über die Art dieser Substanz vorzunehmen und dann namentlich auch Vergleichungen dieses Gehaltes mit demjenigen von Kunsthonigen anzustellen. Zu diesem Zwecke wurden neben Gesamtstickstoffbestimmungen nach dem Verfahren vion $\mathrm{Kjeldahl}$ vorerst Ausfällungen der Albuminate mit verschiedenen Fâllungsmitteln versucht. Auch sind Bestimmungen des Ammoniaks und Amidstickstoffes im Gange, über welche später Mitteilung gemacht werden soll.

1) J. König, Chemie der menschlichen Nahrungs- und Genußmittel. 1, S. 923.

2) W. Bräutigam (Pharm.-Ztg. 1902, 47, 109) fand, daßs Kunstbonige keine Eiweißsreaktion gaben. - Vergl. auch G. Marpmann in Pharm. Ztg. 1908, 48, 1010.

3) Zeitschr. f. physiol. Chem. 1886, 10, 227. 